\title{
Model validation of flow and dispersion around a cube
}

\author{
D.E. Stevens, S.T. Chan, R.L. Lee
}

January 20, 2000

U.S. Department of Energy

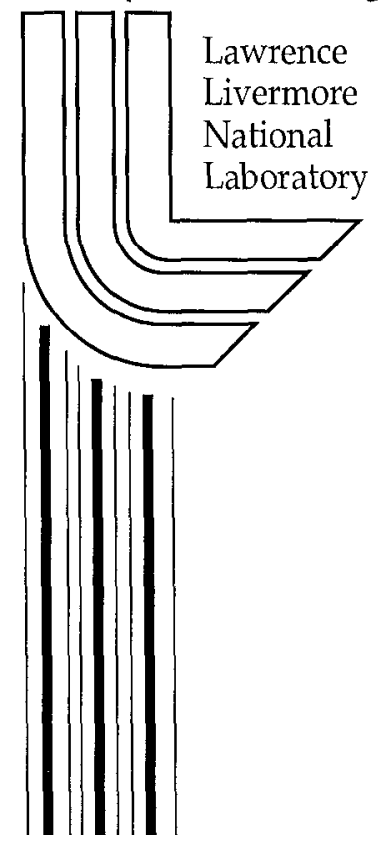




\section{DISCLAIMER}

This document was prepared as an account of work sponsored by an agency of the United States Government. Neither the United States Government nor the University of California nor any of their employees, makes any warranty, express or implied, or assumes any legal liability or responsibility for the accuracy, completeness, or usefulness of any information, apparatus, product, or process disclosed, or represents that its use would not infringe privately owned rights. Reference herein to any specific commercial product, process, or service by trade name, trademark, manufacturer, or otherwise, does not necessarily constitute or imply its endorsement, recommendation, or favoring by the United States Government or the University of California. The views and opinions of authors expressed herein do not necessarily state or reflect those of the United States Government or the University of California, and shall not be used for advertising or product endorsement purposes.

Work performed under the auspices of the U.S. Department of Energy by the University of California Lawrence Iivermore National I aboratory under Contract W-7405-Eng-48.

This report has been reproduced directly from the best available copy.

Available to DOE and DOE contractors from the Office of Scientific and Technical Information

P.O. Box 62, Oak Ridge, TN 37831

Prices available from (423) 576-8401

http://apollo.osti.gov/bridge/

Available to the public from the National Technical Information Service

U.S. Department of Commerce 5285 Port Royal Rd., Springfield, VA 22161 http://www.ntis.gov/

OR

Lawrence Livermore National Laboratory Technical Information Department's Digital Library http://www.llnl.gov/tid/Library.html 


\title{
Model validation of flow and dispersion around a cube
}

\author{
D. E. Stevens, S.T. Chan, and R.L. Lee. \\ Lawrence Livermore National Laboratory, P.O. Box 808, L-103, Livermore, CA 94551
}

\begin{abstract}
This paper compares results for flow over a cube between laboratory experiments and two numerical simulations. One of the simulations is a Reynolds-averaged Navier-Stokes (RANS) calculation, the other a large eddy simulation (LES). Both the structure of the flow and dispersion of a source behind the cube are compared. It was found that both simulations performed well when mean flows are compared. For dispersion, the LES performed better than the RANS simulation in that it was able to capture the effect of vortex shedding and produce a wider dispersion pattern. The plume in the RANS simulation is very similar to instantaneous realizations of the plume in the LES. Near the cube, the results were very similar. This model validation study suggests that the high cost of LES computations may be warranted when detailed time-varying solutions are of high interest. However the high fidelity RANS approach is a cost-effective alternative to LES in obtaining time-average mean field results.
\end{abstract}

\section{Introduction}

The prediction of atmospheric flow and pollutant dispersion in an urban area is difficult due to the presence of buildings and numerous surface-mounted obstacles. Individual buildings exhibit significant geometrical variations with building wakes from adjacent buildings interfering and altering the trajectory of street level plumes. We have developed a numerical model for predicting airflow and pollutant dispersal on building-scales from a single building to multi-building complexes. Model validation is a fundamental component of establishing the credibility of this model for use in the assessment of flow and dispersion of hazardous agents around buildings. It is necessary to establish the credibility of a model on simpler flows, before using a model to assess dispersion involving very complicated flows.

This paper describes a validation of flow and dispersion around a cubical building. There have been several laboratory experiments and many research papers published on this flow (see Lakehal and Rodi (1997), Shah (1998), and Zhang, Arya, and Snyder (1996) for references). This enables us to use this flow as a benchmark for testing model accuracy. The relative simplicity of this flow makes it possible to compare, via non-dimensional parameters, a collection of widely varying model simulations and experiments in a common format.

The fidelity of the model with respect to the flow field is judged by comparison with results from a laboratory experiment by Martinuzzi and Tropea (1993). There the non-dimensional velocity, $U / U_{0}$, where $U$ is the stream-wise horizontal velocity field and $U_{0}$ is the mean inflow 
velocity, provides a useful metric for comparing the winds responsible for dispersion. The degree of variability in the flow can be judged via the normalized turbulent kinetic energy $k / U_{0}^{2}$. Turbulent kinetic energy (TKE) provides an estimate for the turbulent variations that occur in this flow as it is computed as a variance of actual velocities from their time-averaged mean value.

Dispersion of a tracer in the wake of the cube has been much less studied than the flow field itself. However, for release assessment purposes, dispersion is more important than the actual flow field. Here we use a study by Zhang, Arya, and Snyder (1996) who released a ground level tracer $0.25 \mathrm{H}$ downstream from the cube where $H$ is the height of the cube. The experiment showed that the tracer is entrained into the recirculating eddy behind the cube and dispersed downstream. An important feature of the dispersion pattern is that it quickly becomes much wider than the cube width within a few block heights downstream. An explanation of this behavior can be obtained by comparing the LES with RANS results.

The model used in this report is FEM3MP. It is a massively parallel version of the earlier model FEM3CB which is described in: Chan, Lee, and Leone (1999), Chan (1994), and Gresho and Chan (1998). This model is a finite element model with an implicit time integration technique and has options for performing both RANS and LES simulations. This report will only address those aspects of the model which differentiate the RANS from the LES formulation.

\section{Governing Equations}

The equations modeled in this validation study are a subset of the generalized anelastic equations available to FEM3MP. The equations used here are for incompressible flow with an additional advected scalar. The equations, written in Cartesian tensor form, are:

$$
\begin{aligned}
& \frac{\partial u_{i}}{\partial t}+u_{j} \frac{\partial u_{i}}{\partial x_{j}}=-\frac{\partial p}{\partial x_{i}}+\frac{\partial}{\partial x_{j}}\left(-\overline{u_{j}^{\prime} u_{j}^{\prime}}\right) \\
& \frac{\partial u_{j}}{\partial x_{j}}=0 \\
& \frac{\partial c}{\partial t}+u_{j} \frac{\partial c}{\partial x_{j}}=\frac{\partial}{\partial x_{j}}\left(-\overline{u_{j}^{\prime} c^{\prime}}\right)
\end{aligned}
$$

In the above equations, $u_{i}$ is the $i$-th component of the mean velocity, $c$ is the mean tracer concentration, and $p$ is the dynamic pressure used to maintain mass consistency. The momentum and scalar equations have source terms that arise from the turbulence submodel used. The chief difference between the RANS and LES simulations presented are the manner in which the turbulent stress, $\overline{u_{i}^{\prime} u_{j}^{\prime}}$ and scalar flux $\overline{u_{j}^{\prime} c^{\prime}}$ are parameterized by their turbulence models.

RANS modeling parameterizes $\overline{u_{i}^{\prime} u_{j}^{\prime}}$ and $\overline{u_{j}^{\prime} c^{\prime}}$ by using gradient-type terms to represent the whole range of turbulent scales. As a result, the spatial and temporal resolution requirements are often 
less stringent than those of an LES simulation. This makes this type of modeling very attractive for situations that require a large number of simulations and mean quantities are adequate. Its drawback is that it may require a very sophisticated turbulence model and that certain information such as the turbulent variation of the velocities and scalar are available only in a parameterized form.

LES modeling is different in that it seeks to model most of the turbulent variation with the numerics of the resolved motions. A turbulence model is used only to parameterize the motions that are smaller than a cutoff length scale, $l$, typically chosen as a small multiple of the mesh spacing. If most of the turbulence is organized on scales larger than $l$, then a very simple model for the effect of motions smaller than $l$ can be used without compromising accuracy. The drawback of this procedure is that one must often use a large number of timesteps to achieve quasi-steady conditions after which stable means and variances can be computed. Furthermore, the choice of $l$ is often arbitrary, with various researchers siding on the side of small or large values, depending on their optimism and computer resources. The consensus view indicates that in order to capture the bulk of the turbulent variations, overall computational costs are much higher than typically required in a RANS simulation.

The mechanics of simulating dispersion in an LES model are different from a RANS model. A computational advantage of RANS simulation is that given a sufficiently accurate steady-state velocity field, the dispersion results are obtained as a separate calculation from the flow field. These calculations are decoupled as long as there are no physical interactions between the two fields. This is useful for assessment studies involving non-reacting agents, where one might want to run many dispersion calculations assuming different source scenarios for a given wind field. Since this is just a scalar calculation, execution time can be an order magnitude faster than that of the flow (vector) calculation. In an LES model, it is necessary to compute the dispersion calculation the same time as one generates the flow field, since the velocity field is timedependent and it is infeasible to save the velocity field in sufficient detail. This practically limits the scenarios one can generate, since dispersion results cannot be generated from a pre-computed velocity field.

Also, LES has theoretical difficulties if the source is of a short duration. One can try to embed an ensemble of dispersion calculations in an LES by releasing sources of multiple scalars at different times. Unfortunately, this requires the assessor to know all of the desired source parameters at the start of a long simulation. However, dispersion in an LES simulation captures much of the turbulent variation. For completeness, the ideal assessment scenario should include both types of simulation.

The following subsections describe the RANS and LES turbulence models in more detail. 


\subsection{RANS Turbulence Model}

The RANS model used in this report has a nonlinear eddy viscosity (NEV) turbulence submodel developed by Suga (1995). This 3-equation turbulence submodel has many desirable properties, including anisotropy, a cubic constitutive law, and no need for wall functions. It suffers from none of the maladies that normally afflict the commonly used $k-\varepsilon$ turbulence submodel. Additionally, since the model has been derived from the Reynolds stress closure models, it retains many of their attributes-but at a significantly reduced computational cost. This submodel was initially implemented and tested in another time-dependent Navier-Stokes solver, with encouraging results in a simulation of the flow pass an automobile-like body (Gresho and Chan, 1998).

The crux of the submodel involves the following three fairly complex, coupled equations, with details available in Suga (1995), Gresho and Chan (1998):

$$
\begin{aligned}
& \frac{\partial k}{\partial t}+u_{j} \frac{\partial k}{\partial x_{j}}=\frac{\partial}{\partial x_{i}}\left[\left(v \delta_{i j}+0.22 f_{g} \overline{u_{i}^{\prime} u_{j}^{\prime}} \frac{k}{\bar{\varepsilon}}\right) \frac{\partial k}{\partial x_{j}}\right]+P_{K} \pm \varepsilon, \\
& \frac{\partial \bar{\varepsilon}}{\partial t}+u_{j} \frac{\partial \bar{\varepsilon}}{\partial x_{j}}=\frac{\partial}{\partial x_{i}}\left[\left(v \delta_{i j}+0.18 f_{g} \overline{u_{i}^{\prime} u_{j}^{\prime}} \frac{k}{\bar{\varepsilon}}\right) \frac{\partial \bar{\varepsilon}}{\partial x_{j}}\right]+\left(1.2 P_{K} \pm C_{\varepsilon} \bar{\varepsilon}\right) \frac{\bar{\varepsilon}}{k}+P_{\varepsilon} \\
& \frac{\partial A_{2}}{\partial t}+u_{j} \frac{\partial A_{2}}{\partial x_{j}}=\frac{\partial}{\partial x_{i}}\left[\left(v \delta_{i j}+0.22 \overline{f_{g}} \overline{u_{i}^{\prime} u_{j}^{\prime}} \frac{k}{\bar{\varepsilon}}\right) \frac{\partial A_{2}}{\partial x_{j}}\right]+\frac{a_{i j}}{k}\left[P_{i j} \pm \varepsilon_{i j}+\phi_{i j}\right] \pm \frac{2 A_{2}}{k} P_{K} .
\end{aligned}
$$

In the above equations, $k$ is the turbulent kinetic energy (TKE), $\bar{\varepsilon}$ is the isotropic dissipation rate, and $A_{2}$ is the second invariant of the dimensionless anisotropic Reynolds stress tensor.

The Reynolds stresses are defined by a cubic constitutive relationship

$$
\begin{aligned}
\overline{u_{i}^{\prime} u_{j}^{\prime}} & =\frac{2}{3} k \delta_{i j} \pm v_{T} S_{i j}+\frac{v_{T} k}{\bar{\varepsilon}}\left\{c_{1}\left(S_{i k} S_{k j} \pm \frac{1}{3} S_{k \ell} S_{k \ell} \delta_{i j}\right)\right. \\
& +c_{2}\left(\Omega_{i k} S_{k j}+\Omega_{j k} S_{k i}\right)+c_{3}\left(\Omega_{i k} \Omega_{j k} \pm \frac{1}{3} \Omega_{\ell k} \Omega_{\ell k} \delta_{i j}\right) \\
& \left.+\frac{k}{\bar{\varepsilon}}\left[c_{4}\left(S_{k i} \Omega_{\ell j}+S_{k j} \Omega_{\ell i}\right) S_{k \ell}+c_{5} S_{i j} S_{k \ell} S_{k \ell}\right]+c_{6} S_{i j} \Omega_{k l} \Omega_{k \ell}\right\},
\end{aligned}
$$

in where $S_{i j}$ is the mean strain rate tensor and $\Omega_{i j}$ is the mean rotation tensor. The isotropic turbulent eddy viscosily is defined as

$$
v_{t}=c_{\mu} f_{\mu} k^{2} / \bar{\varepsilon}
$$

where $c_{\mu}$ is a turbulent viscosity parameter and $f_{\mu}$ is a wall damping function. 


\subsection{LES sub-grid turbulence model}

The LES turbulence model is simpler than the RANS three-equation model. The sole role of the turbulence model in an LES simulation is to parameterize the effect of motions that cannot be resolved by the computational mesh. The simplest such parameterization and the one used here was developed by Smagorinsky (1963) and Lilly (1962). It assumes that the subgrid TKE, $e$, is locally in equilibrium between the locally generated shear production and dissipation. There are more complicated subgrid models such as the one by Deardorff (1974), where this assumption is relaxed and a subgrid kinetic energy is advected in a manner similar to the TKE in the above threeequation model. In the Smagorinsky turbulence model, the subgrid TKE is found directly from the equilibrium assumption, which can be manipulated to yield

$$
e^{1 / 2}=\frac{c_{S}^{2}}{c_{m}}\left|S_{i j}\right|,
$$

which yields the eddy viscosity,

$$
K_{m}=c_{S}^{2} \cap\left|S_{i j \mid}\right|
$$

Here $c_{S}$ and $c_{m}$ are empirical constants with values of 0.1 and 0.3 in this study. The subgrid scale fluxes are then found from a simple down-gradient mixing-length relationship between velocity and scalar gradients and the subgrid stress and fluxes

$$
\begin{aligned}
& \overline{u_{i}^{\prime} u_{j}^{\prime}}= \pm K_{m} S_{i j}, \\
& \overline{u_{i}^{\prime} c^{\prime}}= \pm K_{m} \frac{\partial c}{\partial x_{j}},
\end{aligned}
$$

Although simpler conceptually, the properties of the flow are more difficult to diagnose for a LES than a RANS simulation. It often requires running the simulation for a very long time and using temporal averaging to generate mean statistics. This is illustrated by the following comparison of how TKE is generated in RANS versus LES. For the RANS calculation, TKE is computed explicitly and can be used as a scalar diagnostic. For the LES, one must first generate a mean velocity field, and the deviations from this field, square and average again to generate the resolved TKE. Since this is a statistical quantity, a sufficiently long averaging period must be used to generate stable statistics. Averaging is also needed for the sub-grid TKE, since the sum of these two quantities has to be used for comparison with RANS and the experimental results.

LES subgrid models have similar computational problems as RANS turbulence models. One problem associated with these turbulence models is their ability to model near wall behavior. For this study, we used a simulation with no-slip boundary conditions on the bottom boundary for the RANS, and free slip for the LES. This maximized turbulent dispersion in the LES. Another factor which maximized turbulent dispersion was using a Smagorinsky coefficient, $c_{s}=0.1$ which 
is a value typically used in engineering studies. This contrasts with the value of $c_{s}=0.23$, a value typically used in atmospheric LES, that yielded less crosswind dispersion.

\section{Results}

Both the RANS and LES used the same computational domain. The height of the cube, $H$, is 1.0 , the channel height is $2 \mathrm{H}$, the width is $7 \mathrm{H}$ and the length of the channel is $10 \mathrm{H}$. The origin is situated at the center of the cube, with the inflow at $\mathrm{x}=-3.5 H$. The flow domain is illustrated in Figure 1. This figure displays the cube and several vertical and horizontal lines where profiles from both the dispersion experiments of Zhang and Snyder (1996) and flow experiments of Martinuzzi and Tropea (1993) are to be presented. Also, shown on this diagram are three planes that are used to compare the RANS simulation versus the LES. These are the symmetry plane along the middle of the domain, the floor plane at the bottom, and the outflow plane where the tracer leaves the domain.

Since the RANS assumes a steady state solution and the geometry was symmetric, only one half the domain was simulated. This enabled the RANS to use a small mesh of $96 \times 37 \times 30$ nodal points. Overall, the simulation took 25 hours on 2-3 processors of a shared multi-processor DEC ALPHA to generate 1200 timesteps. This simulation used as inflow, the mean velocity of a channel flow, with a bulk average velocity of $0.6 \mathrm{~m} / \mathrm{s}$.

The LES used a computational mesh of $129 \times 65 \times 65$ nodal points over 1920 timesteps. This generated a time series of data 57.6 nondimensional time units long, $t^{*}=U_{0} t / H$, where $t^{*}$ the non-dimensional time. A uniform inflow velocity of $0.6 \mathrm{~m}$ was used which matches the inflow characteristics of Zhang, Arya, and Snyder (1996). The LES calculation requires roughly an order of magnitude more effort than the RANS simulation. However, due to the use of parallel computing on 64 processors, this simulation took only 18 hours to generate. Without the use of parallel computing, this simulation would have been much more demanding to perform. It should be noted that the processors on the IBM parallel machine are PowerPC chips with a performance of around $40 \%$ of the DEC ALPHA. Taking this into account yields similar performance in integrated gridpoints per cpu-second.

The timc averaging in this report was done by averaging snapshots of data along, the symmetry, floor, and outflow planes. The model was integrated 40 non-dimensional time units before averaging to avoid incorporating any of the transient from the model initialization. At this point, the mean concentration in the domain had stabilized and the flow has settled down alternately shedding pairs of vortices behind the cube. The averaging period in all the mean LES fields presented here used the last third of the simulation.

Time histories from the LES complement the assessment capabilities of a combined LES and RANS approach. Figure 2 shows a time history of concentration at the surface at $x=3.5 H$. The fundamental period of the simulation is that of the alternately shedding vortices. From Shah 
(1998), a pair of alternating vortices is shed every 8.5 non-dimensional time units. This is equivalent to a Strouhal Number of $S t=H /\left(U_{0} t\right)=0.11$. For our simulation, the Strouhal number is 0.13 which is equivalent to a pair of vortices every 7.5 time units. The estimated period is twice that in this figure as each vortex of the pair advects part of its peak concentration over the centerline. Note there is a strong peak where a concentrated puff of tracer hit this station. While this is not indicative of the mean concentration, a long enough time series can be used to measure the transient phenomena that are responsible for peak exposures.

Figures $3 \mathrm{~A}$ and $3 \mathrm{~B}$ compare the total turbulent kinetic energy at the symmetry plane, Figure $3 \mathrm{~A}$ compares our LES and RANS results with those of Shah's LES. Figure 3B shows the streamlines from Martinuzzi and Tropea, Shah's LES, FEM3MP LES, and RANS. The TKE in the LES tends to be larger and extend closer to the surface than in the RANS. The features of the two LES results are very similar. Our LES tends to predict more TKE behind the cube, while Shah's has a bigger region of large TKE values in front of the cube. The main differences between the two LES models is probably the different boundary conditions used at the top and bottom of the domain. Shah uses no-slip boundary conditions at top and bottom, while we use free-slip boundary conditions to better match the experimental results of Zhang, Arya, and Snyder (1996). The main features of all flow fields are similar. They include the separation zones in front of the cube, on the roof and the two sides, a primary recirculation zone in the wake, and a pair of counterrotating vortices on the horizontal plane (not shown). The reattachment length predicted by the 3-equation NEV turbulence submodel is 1.85 , which agrees well with the value of 1.68 measured by Martinuzzi and Tropea (1993), and with the value of 1.64 predicted by Shah's (1999) LES. The recirculation length of this LES simuation was 1.55 . These lengths compare more favorably than that predicted by the simpler, but widely used standard $k-\varepsilon$ turbulence model results that tend to be around 2.85 (Chan 1999), which is much larger than all above values.

In Figures 4 and 5, we compare the predicted turbulent kinetic energy and velocity fields with the data of Martinuzzi and Tropea (1993). The features of the LES agree better with the experimental data. The small discrepancies at the top of the domain are generated by the free slip boundary conditions used in the LES model to approximate the higher top boundary location of the Zhang, Arya, and Snyder (1996) experiment. The RANS velocities and the LES velocities agree with the observational data in that they predict all of the bulk flow features observed in the experiment. However, the LES predicts the magnitudes of TKE better than the RANS as the RANS under-predicts the TKE near the peaks. Both model results predict peaks of TKE at approximately the correct locations.

The results of Zhang, Arya, and Snyder (1996) are used to evaluate the accuracy of concentration patterns from the model. These results where created with a tow tank in which a cube mounted to a plate was moved at $0.6 \mathrm{~m} / \mathrm{s}$ through a water tank 18 meters long. The cube was $0.1 \mathrm{~m}$ high and the tank was $1 \mathrm{~m}$ wide by $1 \mathrm{~m}$. All of the turbulence in the experiment was generated by the passage of the cube. The dispersion in their experiments is generated by a small ground-level continuous source $0.25 \mathrm{H}$ centered behind the rear of the cube. The green lines of Figure 1 are the locations where the concentrations downstream from the cube have been measured. 


\section{Summary}

We have presented a validation for our model involving flow and dispersion over a cube. It was found that the LES yields better results but with roughly an order of magnitude increase in cost. The RANS is attractive, if one takes into account the finer grid and smaller timesteps needed for LES as well as the ability of the RANS to decouple dispersion simulations from those of the mean flow. A summary of our comparison with experiment follows:

1. The RANS and LES have similar mean velocity fields near the cube. If mean velocity fields are sufficient and decoupled from the advected scalar species, one can run the dispersion offline using mean data and the RANS solution is the more economical method.

2. The LES captures turbulent variations that the RANS does not model. Hence if LES dispersion is calculated in a coupled manner along with the flow, the results incorporate the effects of transitory eddies. For situations in which the source lasts sufficiently long, LES simulates more accurately the width of the dispersion, whereas RANS slightly under-predicts it. This is useful in situations where the knowledge of trace amounts of advected species is important.

3. Comparisons of instantaneous snapshots of the LES have similar vertical depth and width as the RANS. This indicates the RANS is producing a reasonable plume, but without the spatial fluctuation associated with vortex shedding in the experimentally observed wake.

Finally, this report demonstrates the utility of having both a tested RANS and LES capability in the same modeling framework. We are further validating the accuracy of this framework with both wind tunnel and field experiments.

\section{References}

Chan, S., FEM3C-An improved three-dimensional heavy-gas dispersion model: User's Manual, UCRL-MA-1 16567 Rev. 1, Lawrence Livermore National Laboratory, Livermore, CA, 1994.

Chan, S. T., Case study of flow and dispersion around a cubical building, DARPA report, 1999.

Chan, S. T., R. L. Lee, and J. M. Leone, FEM3CB modeling of turbulence, aerosols and UV degradation, DARPA report, 1999.

Deardorff, J. W., The use of subgrid transport equations in a three-dimensional model of atmospheric turbulence. J. Fluids Eng., 95, 429-438, 1973.

Gresho, P. and S. Chan, Projection 2 goes turbulent-and fully implicit, Int. J. Comp. Fluid Dyn., 9, 249-272, 1998.

Lakehal, D. and W. Rodi, Calculation of the flow past a surface-mounted cube with two-layer turbulence models, $J$ Wind Eng. Ind. Aerodyn., 67\&68, 65-78, 1997.

Lilly, D. K., On the numerical simulation of buoyant convection. Tellus, 14, 148-171, 1962.

Martinuzzi, R. and C. Tropea, The flow around surface-mounted, prismatic obstacles placcd in a fully developed channel flow, J. Fluids Eng., 115, 85-92, 1993. 
Shah, K.B., Large eddy simulations of flow past a cubic obstacle, Ph.D. dissertation, Stanford University, June 1998.

Smagorinsky, J., General circulation experiments with the primitive equations: 1. The basic experiment., Mon. Wea. Rev., 121, 1847-1859, 1963.

Suga, K., Development and application of a nonlinear eddy viscosity model sensitized to stress and strain invariants. Ph.D. thesis, UMIST, Manchester, UK, 1995.

Zhang, Y., S. Arya, and W. Snyder, A comparison of numerical and physical modeling of stable atmospheric flow and dispersion around a cubical building, Atm.Env. 30, 1327-1345, 1996. 


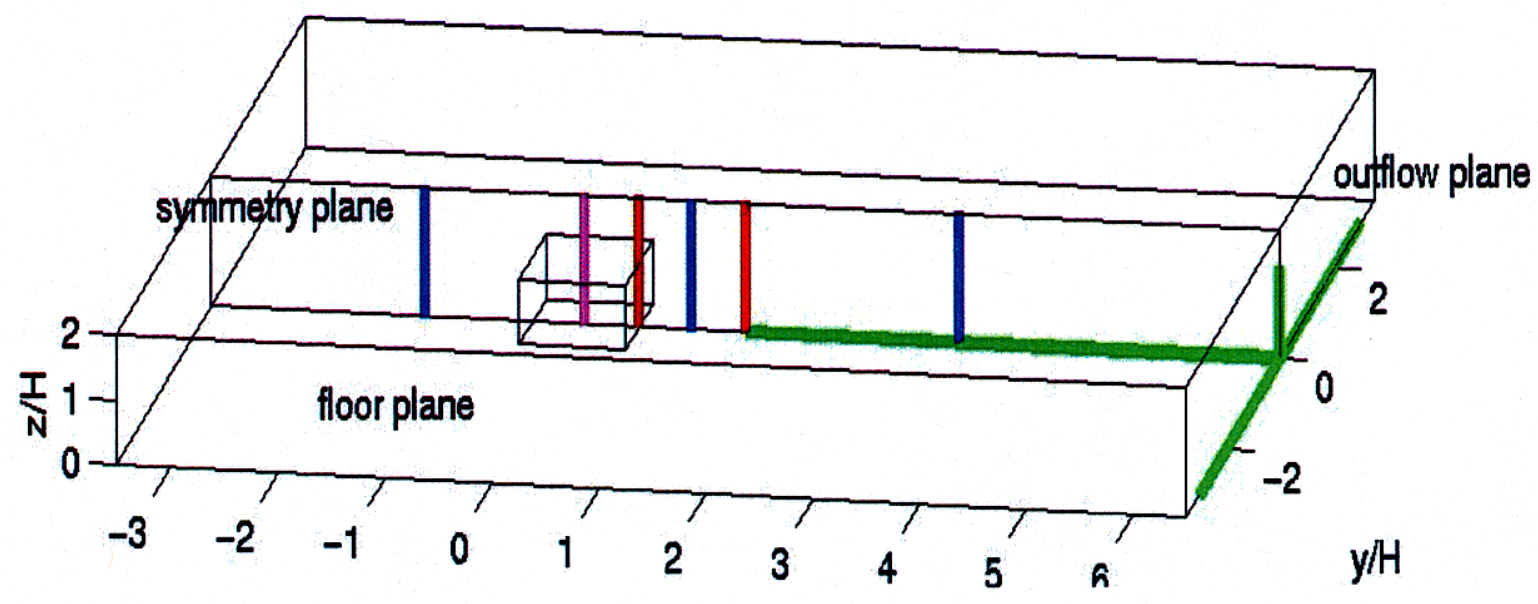

Figure 1: Computational domain, experimental results for the tracer are plotted along green lines, velocity along blue lines, TKE along red lines, and along the magenta line both TKE and momentum.

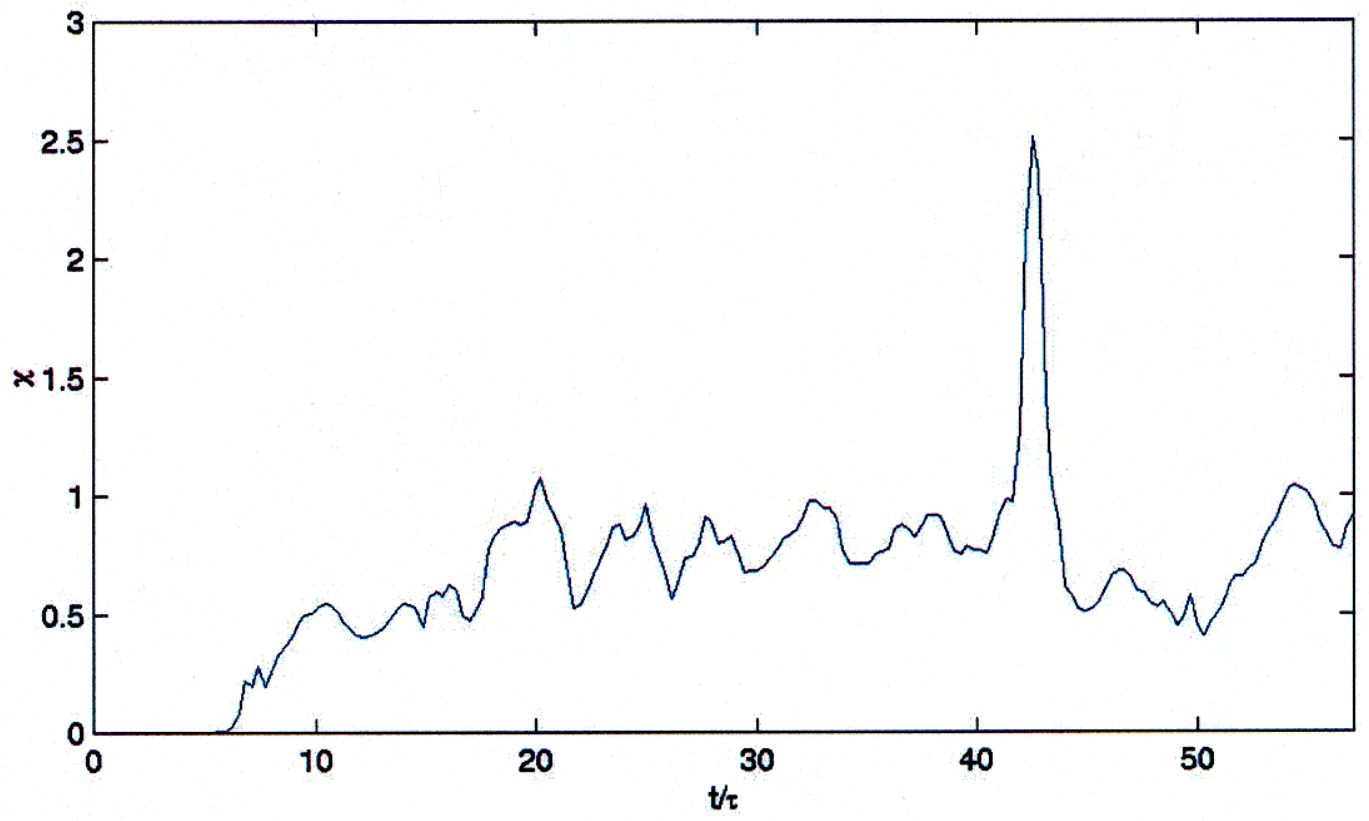

Figure 2: Time history of concentration at $\mathrm{x}=3.5 \mathrm{H}, \mathrm{y}=0.0, \mathrm{z}=0.05 \mathrm{H}$. 

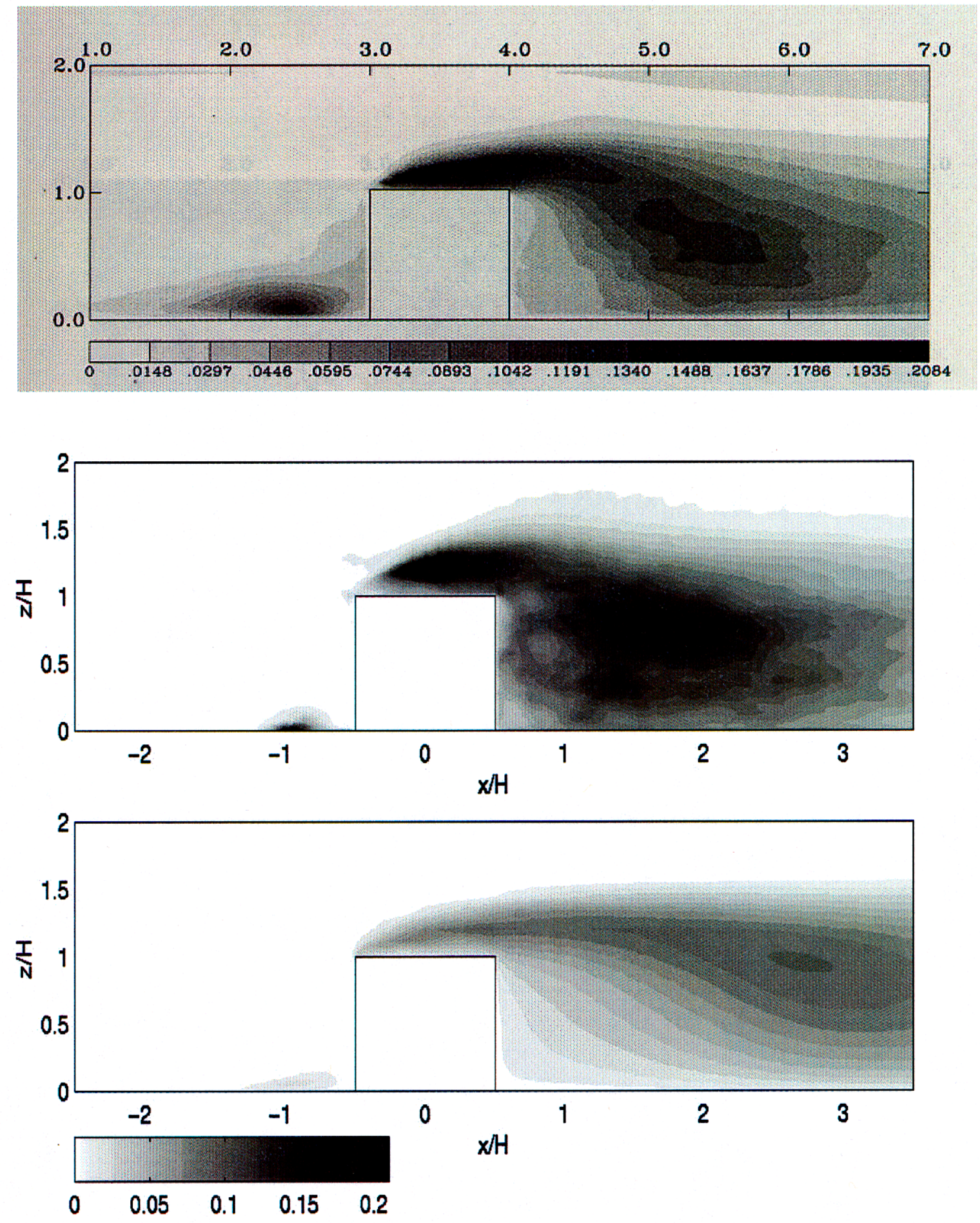

Figure 3A: Comparison of TKE along the symmetry plane for Shah's LES(top), FEM3MP LES (middle) and RANS (bottom). 

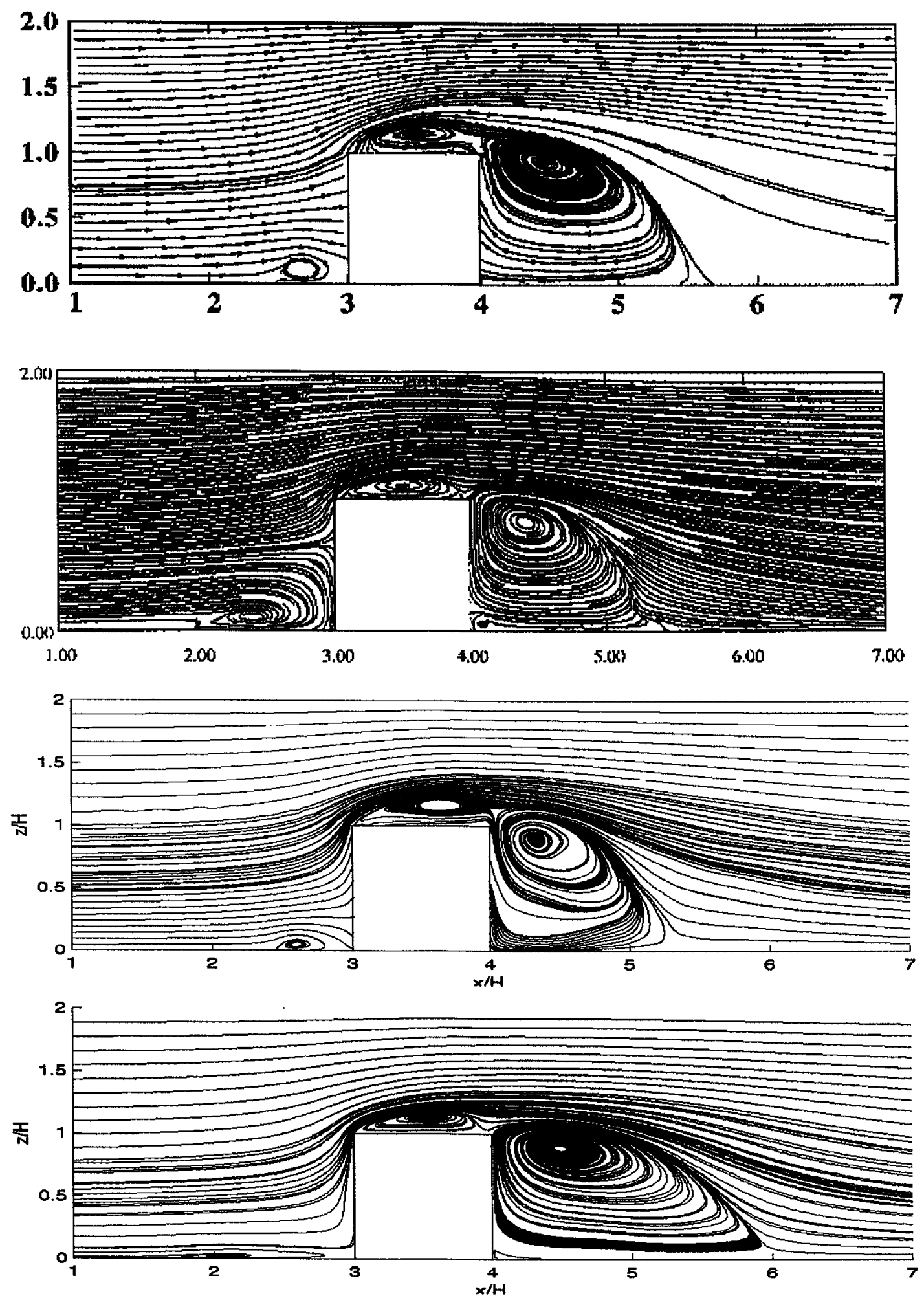

Figure 3B: Comparison of streamlines along the symmetry plane for the experimental results of Martinuzzi and Tropea (top), Shah (top middle), FEM3MP LES (bottom middle) and RANS (bottom). 

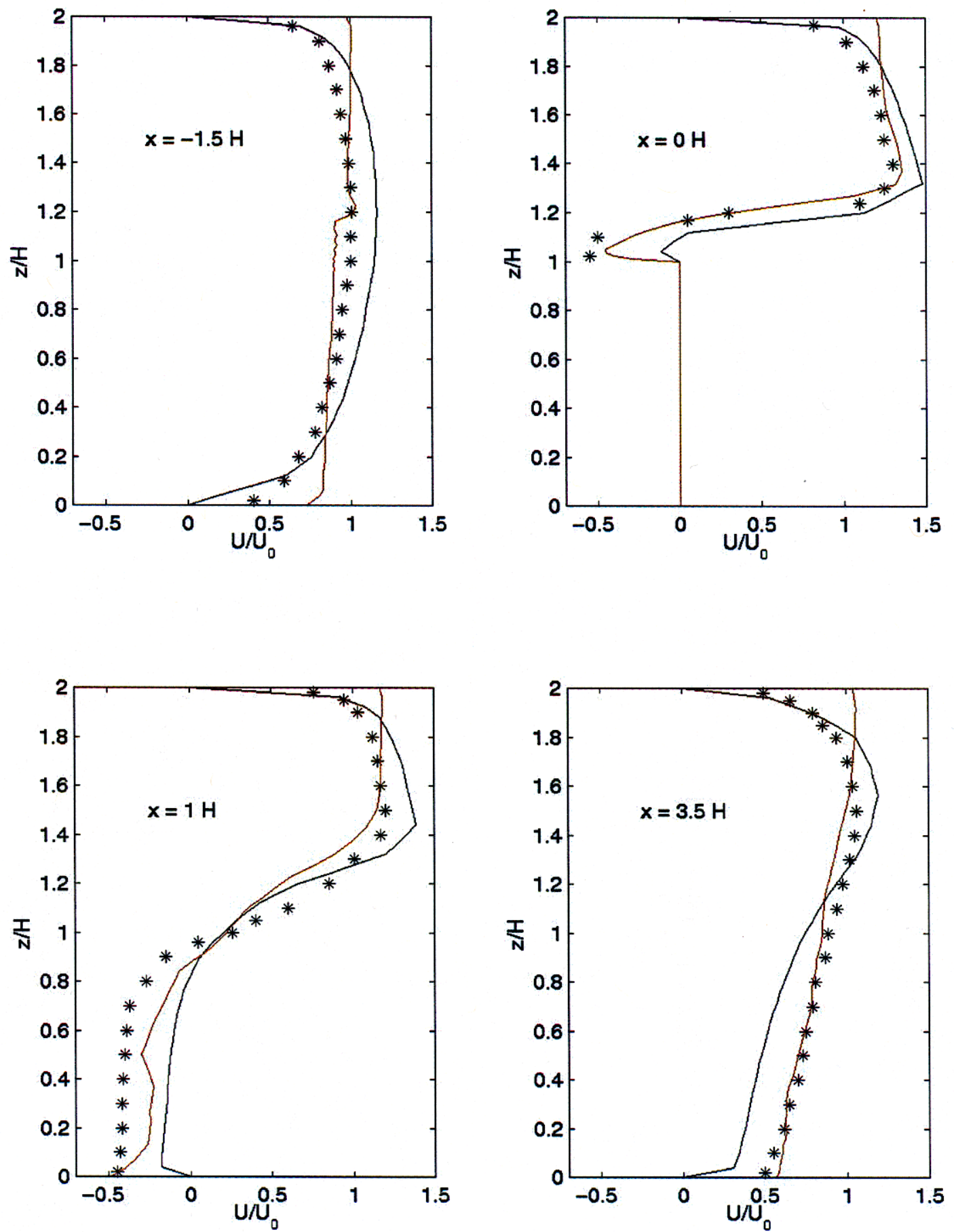

Figure 4: Comparison of the mean stream-wise velocity for experiment (dots), LES (red line) and RANS (black line) at four vertical profiles along the symmetry plane. 

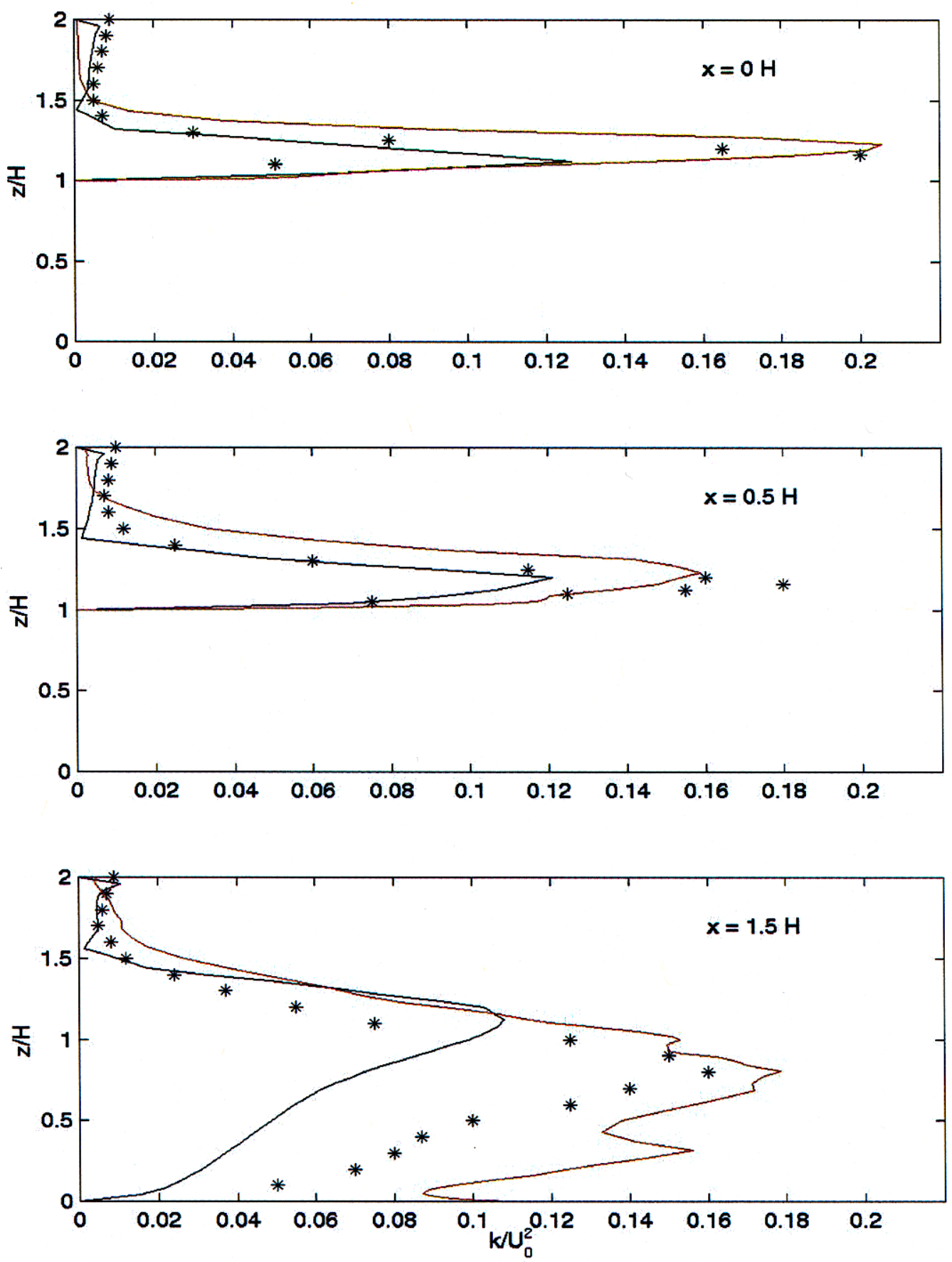

Figure 5: Comparison of the TKE for experiment (dots), LES (red line) and RANS (black line) at three vertical profiles along the symmetry plane. 

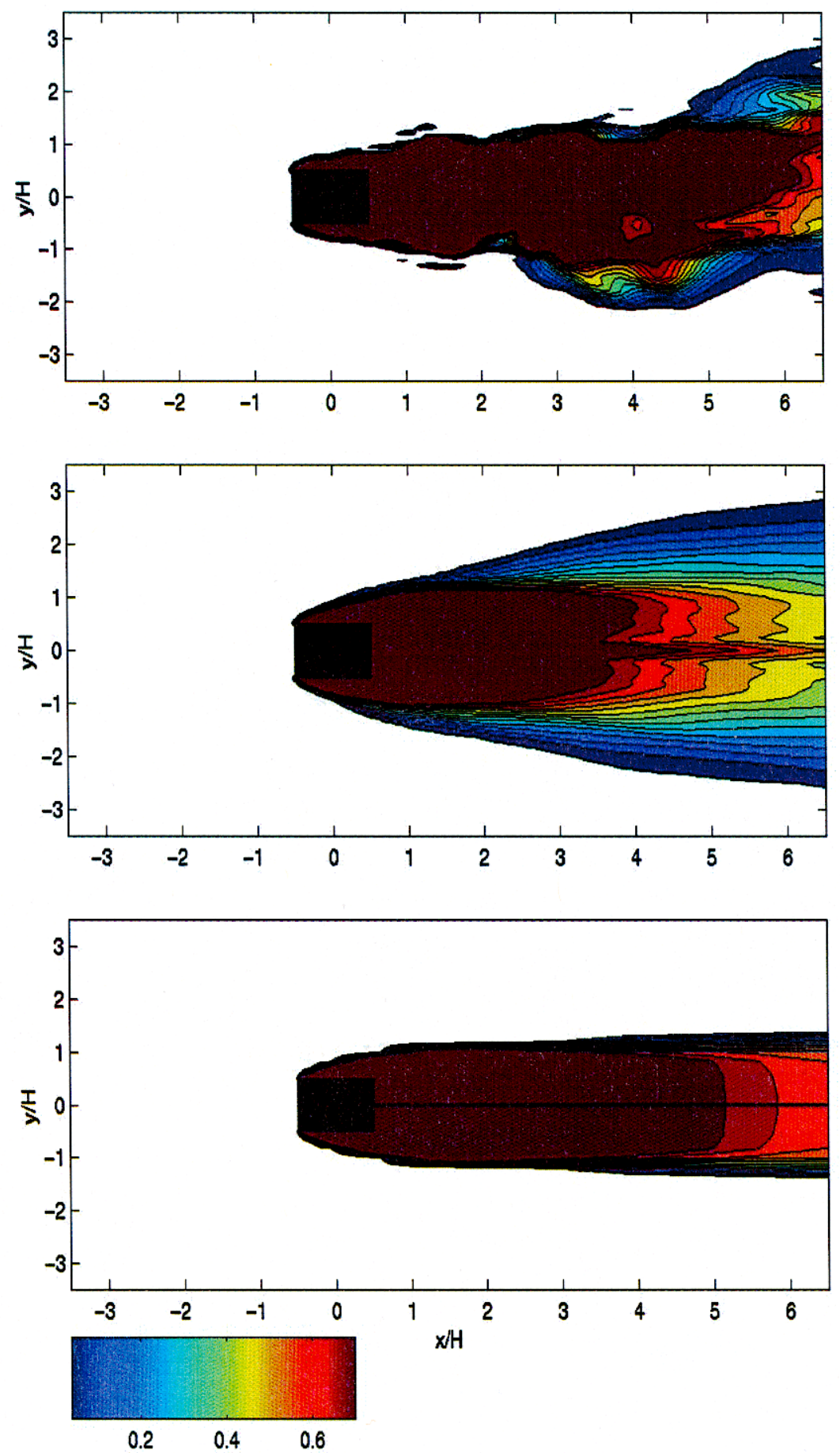

Figure 6: Tracer concentrations along the floor plane showing the instantaneous pattern from the LES (top), the mean from the LES simulation (middle), and from the RANS simulation (bottom). 

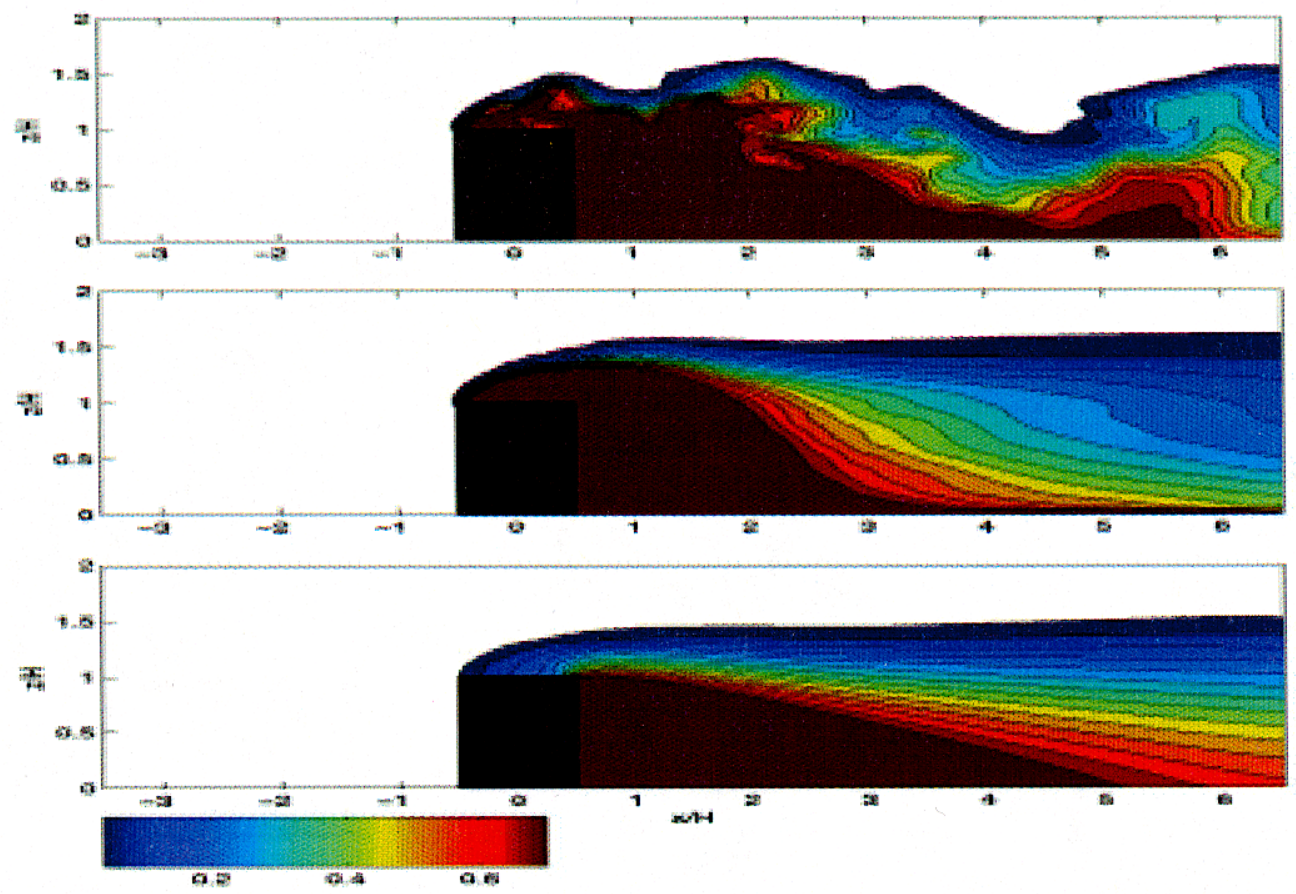

Figure 7: Tracer concentrations along the symmetry plane showing the instantaneous pattern from the LES (top), the mean from the LES simulation (middle), and from the RANS simulation (bottom).
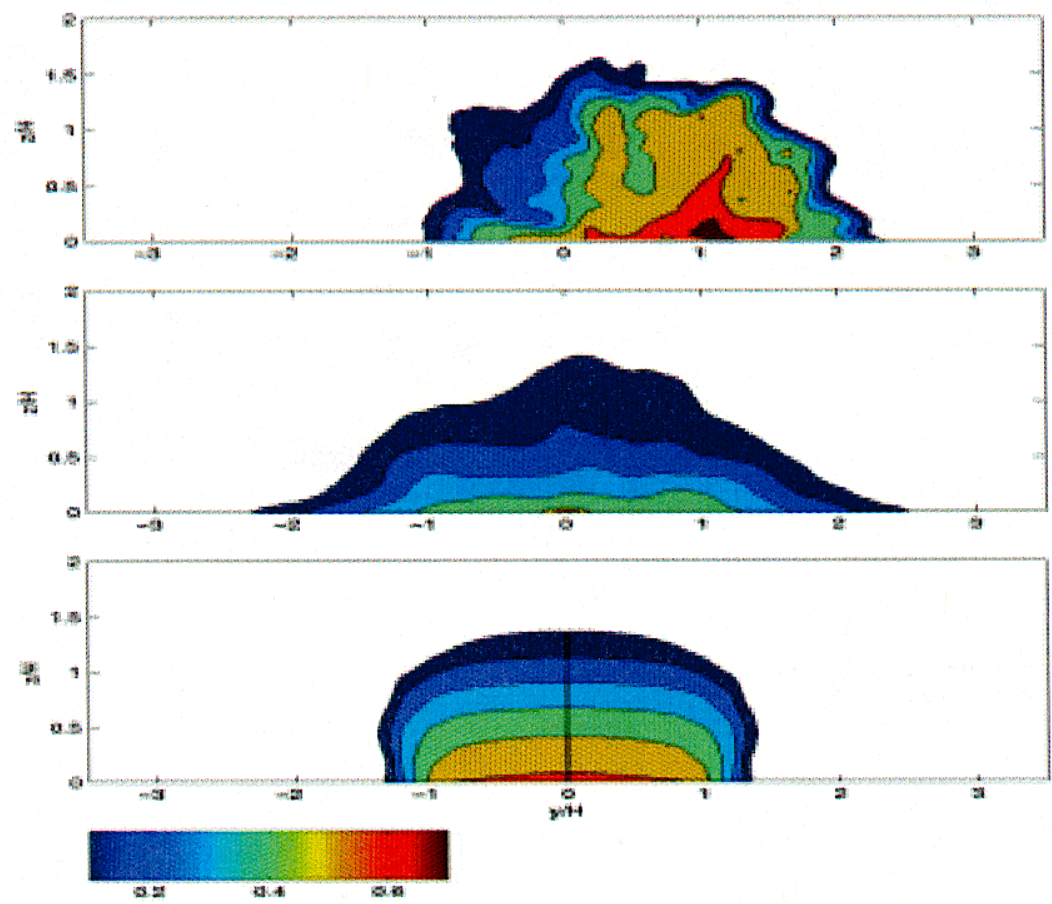

Figure 8: Tracer Concentration at the outflow plane showing the instantaneous pattern from the LES (top), the mean from the LES simulation (middle), and from the RANS simulation (bottom). 

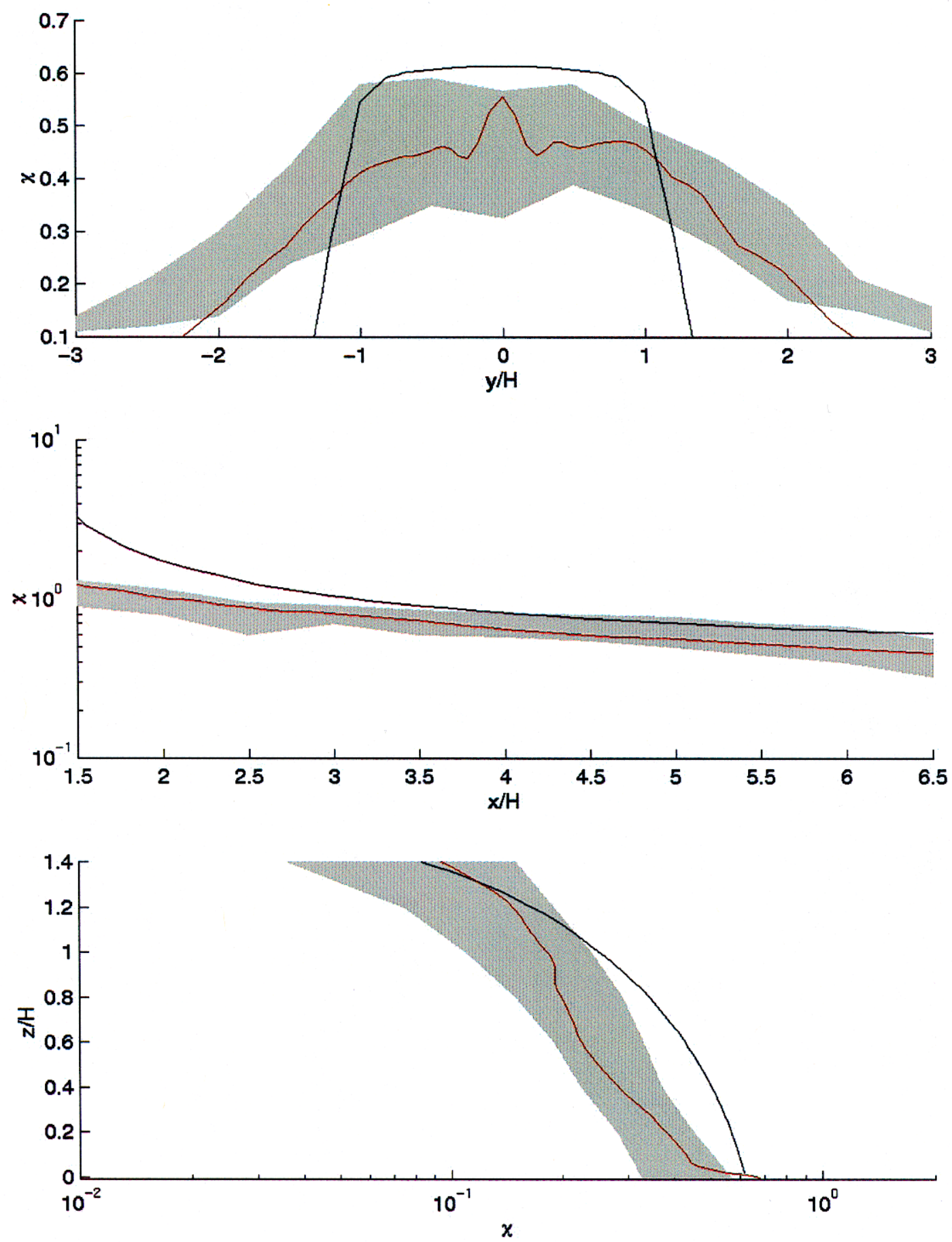

Figure 9: Comparison of the mean concentrations along the green lines of Figure 1. (top) the horizontal line at outflow, (middle) the horizontal line at the bottom of the symmetry plane, (bottom) the vertical line at outflow. 